\title{
Status of the CMS detector and upgrade plans
}

\section{Luigi GUIDUCCI for the CMS Collaboration*}

Università degli Studi di Bologna and INFN

E-mail: guiducci@bo.infn.it

The CMS experiment at the LHC collected $5.5 \mathrm{fb}^{-1}$ of proton proton collisions data at a center of mass energy of $7 \mathrm{TeV}$ in 2011 and more than $20 \mathrm{fb}^{-1}$ at $8 \mathrm{TeV}$ energy in 2012. The CMS detector has shown excellent performance and very good data taking efficiency. The operational experience will be discussed focusing on relevant technical aspects. The performance of CMS subdetectors will be illustrated. Emphasis will be put on the solutions adopted during 2012 run to adapt to the increase in luminosity of the LHC while mantaining the high quality of the physics objects delivered to offline analysis. New challenges, dictated by future LHC luminosity scenarios, are ahead of CMS: an overview of the detector upgrade plans, both on medium and long term range, will be given.

LHC on the March

20-22 November 2012

Institute for High Energy Physics, Protvino,Moscow region, Russia

\footnotetext{
*Speaker.
} 


\section{The CMS detector at the $\mathrm{LHC}$}

The central feature of the Compact Muon Solenoid (CMS) apparatus is a superconducting solenoid of $6 \mathrm{~m}$ internal diameter, providing a magnetic field of $3.8 \mathrm{~T}$. Within the superconducting solenoid volume are a silicon pixel and strip tracker, a lead tungstate crystal electromagnetic calorimeter (ECAL), and a brass/scintillator hadron calorimeter (HCAL). Muons are measured in gas-ionization detectors embedded in the steel return yoke outside the solenoid. Three different type of detectors are used: Drift Tubes (DT), Cathode Strip Chambers (CSC) and Resistive Plate Chambers (RPC). Extensive forward calorimetry complements the coverage provided by the barrel and endcap detectors. A more detailed description can be found in Ref. [1].

\section{CMS data taking during LHC Run 1}

\subsection{Luminosity, efficiency and data taking conditions}

At the end of 2011, the LHC had delivered more than $6 \mathrm{fb}^{-1}$ of proton-proton collisions at a center of mass energy of $7 \mathrm{TeV}$. During the 2011-2012 winter stop, interventions to the machine allowed higher beam energy $(4+4 \mathrm{TeV})$ and higher peak luminosity $\left(7.5 \times 10^{33} \mathrm{~cm}^{-2} \mathrm{~s}^{-1}\right)$. Thanks to the excellent performance of the machine an integrated luminosity of $23.3 \mathrm{fb}^{-1}$ was delivered to CMS in 2012. The luminosity delivered by the LHC is shown in Fig. 1 as a function of time, in blue. In the same figure, the recorded luminosity is shown in yellow. The recording efficiency for the whole 2012 p-p run was $93.5 \%$. The data taking inefficiency was due for about $3 \%$ to (trigger/DAQ) deadtime and the remaining part to downtime events, such as infrastructure or subdetector failures.

The CMS detector, mostly not accessible for maintenance since 2009, have demonstrated a very good reliability. An overview of the fraction of active channels in each subdetector as of end of 2012 is shown in Fig. 1 (right).

The LHC stored 1380 proton bunches per beam, approximately half the number foreseen by the design, running at a bunch spacing of $50 \mathrm{~ns}$ instead of $25 \mathrm{~ns}$. The $\beta^{\star}$ was decreased from
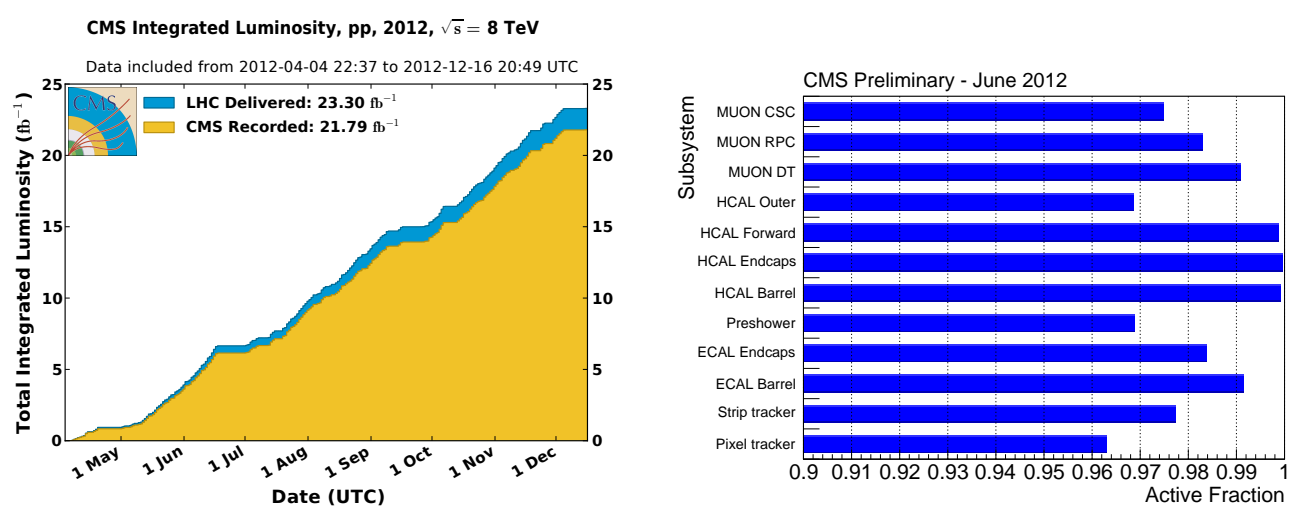

Figure 1: The delivered (blue) and recorded (yellow) luminosity during 2012 p-p run, is shown in the left plot as a function of time. In the right plot, the fraction of active channels in CMS subdetectors during 2012 data taking is shown. 
$\beta^{\star}=1 \mathrm{~m}$ to $\beta^{\star}=0.6 \mathrm{~m}$, obtaining a larger luminosity per bunch pair. The pile-up changed from a design value of $\langle\mu\rangle \sim 20$ to 2012 accelerator fills starting at $\langle\mu\rangle>30$. These conditions represented new and significant challenges to the experiment, in particular for the inner tracking system and for the calorimetry. In the following sections, an overview of the performance of CMS subdetectors is given, with emphasis on pile-up challenges and solutions.

\section{Performance of CMS subdetectors in high pileup environment}

\subsection{Trigger system}

One of the challenges CMS had to face was to keep the trigger thresholds low as pileup increased. CMS trigger system is based on two levels: the Level-1 Trigger [2] is a dead time free, fixed latency hardware system aimed at the identification and fast measurement of muons, electron/photon candidates, jets and energy sums. The Level-1 Trigger system selects events at a rate below $100 \mathrm{kHz}$, at which the detector is fully read out. The High Level Trigger [3], implemented as dedicated software running on a farm of commercial CPUs, performs event building and lowers the rate to storage down to several hundred $\mathrm{Hz}$.

As an example of Level-1 Trigger operations, in Fig. 2 (left) the Level-1 Trigger rate vs time for a typical 2012 fill is shown. The discontinuity in the rate corresponds to the change of the set of prescales applied to low threshold and commissioning triggers.
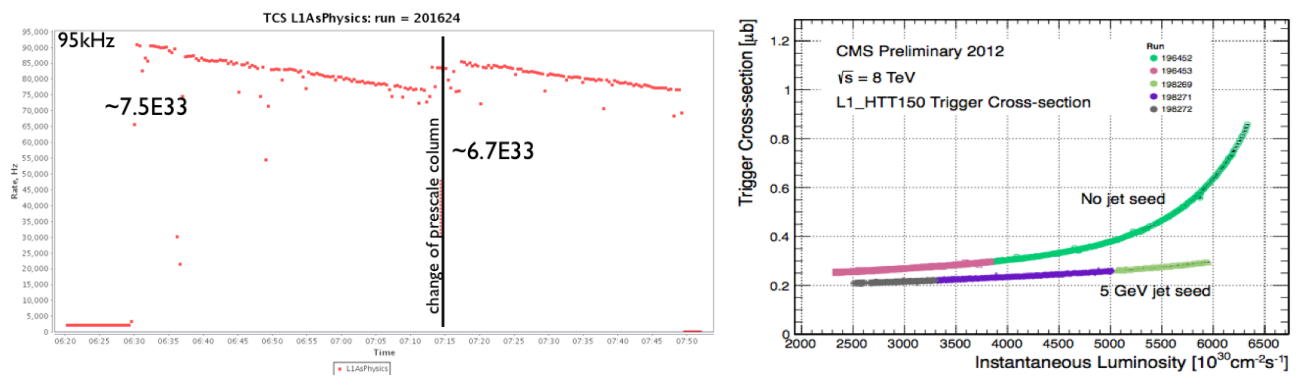

Figure 2: Left: Level-1 Trigger rate as a function of time, for a typical LHC fill. Right: cross-section versus instantaneous luminosity for the L1-HTT-150 trigger (see text for detailed explanation).

The calorimetry at the Level 1 Trigger can be very sensitive to event pile-up: energy from particles produced in different collisions is summed into calorimeter towers, and no tracking in the inner detectors can be used to distinguish between different primary interactions. As a result, the performance of e.g. jet identification and transverse energy measurement algorithms can be modified. As an example, in Fig. 2 (right) the measured cross-section of an energy-sum trigger is shown versus the instantaneous luminosity. This trigger requires the sum of the transverse energy $\left(E_{T}\right)$ of all jets which have $E_{T}>10 \mathrm{GeV}$ and $|\eta|<3$ to be larger than $150 \mathrm{GeV}$. The cross-section of such trigger has a large increase at high luminosity (data with label "No jet seed") as many low $E_{T}$ jets are reconstructed from the energy deposit of pileup events. Thus, a further constraint was introduced into the jet identification algorithm, requiring at least one of the calorimeter towers used to build a jet candidate to have an energy measurement above $5 \mathrm{GeV}$ (shown with label " $5 \mathrm{GeV} \mathrm{jet}$ seed"). With the introduction of this additional cut, the cross-section becomes nearly constant with 
respect to large changes of pileup conditions, allowing the rates to be back under control with no need to rise the energy thresholds, at the cost of a small inefficiency.

At the High Level Trigger, a significant improvement of the trigger performances has been achieved integrating the progress made in the offline software, and exploiting the key features of the detector, like the high magnetic field and the excellent tracking performances, described in the next section.

\subsection{Tracking performance}

Tracking particles in the presence of high pileup is a difficult task, but correct reconstructions of events with up to 40 interactions has been achieved. This is allowed by the excellent resolution of the pixel and silicon strip detectors, below $20 \mu \mathrm{m}$ [6] . The resolution for pixel hits is shown in Fig. 3, left, while on the right side the number of HLT reconstructed pixel vertices versus the number of interactions per crossing is shown: no deviation from linearity is observed. Pixel tracks are essential to perform fast seeding of the full tracking at the HLT, to reconstruct individual vertices and thus to reduce the combinatorial due to pileup. The performances of the tracking algorithm used in HLT are also critical, with respect to offline reconstruction, in terms of memory and CPU; the average processing time per event at the HLT was shown to increase linearly as a function of the instantaneous luminosity.
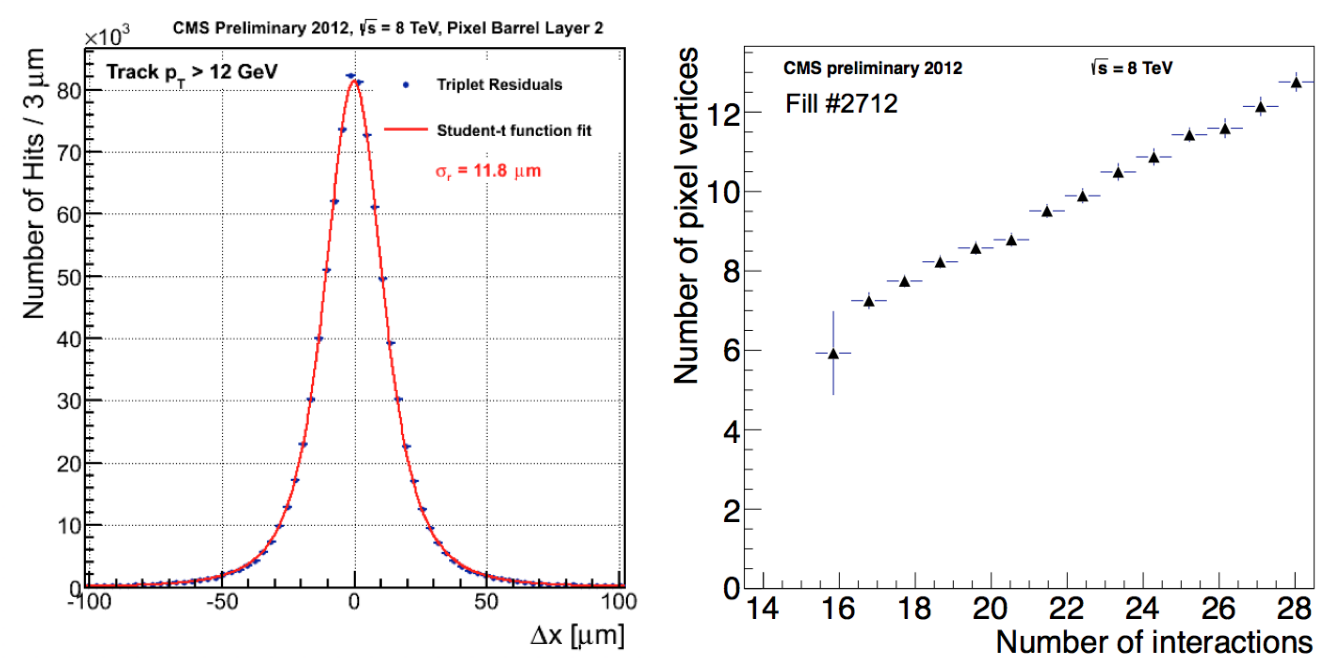

Figure 3: Left: pixel hit resolution determined from data. Right: number of pixel vertices reconstructed by the High Level Trigger versus the number of interactions in the event as determined by the online luminosity measurement.

\subsection{Electromagnetic calorimeter}

The Electromagnetic Calorimeter (ECAL) is a crucial component for many physics analyses, and it is extremely important to have a very good resolution and a stable energy scale. A Laser Monitoring system allows the change of transparency of ECAL crystals to be corrected [4]. The results of such procedure for the barrel region are shown in Fig. 4 (left) where the energy measured 
in ECAL is compared to the momentum measured in the inner tracking system usign $W \rightarrow e v$ events. The result is an RMS of $0.09 \%$ and $0.28 \%$ in the barrel and endcap, respectively. In Fig. 4, right, the resulting impact on the $Z \rightarrow e e$ energy scale and resolution is shown: light blue curve corresponds to uncalibrated data, the red one to data with crystal intercalibration applied, and the dark blue to fully corrected (intercalibration and laser monitoring) data. A mass resolution of about $1.5 \mathrm{GeV}$ is obtained in the barrel region.
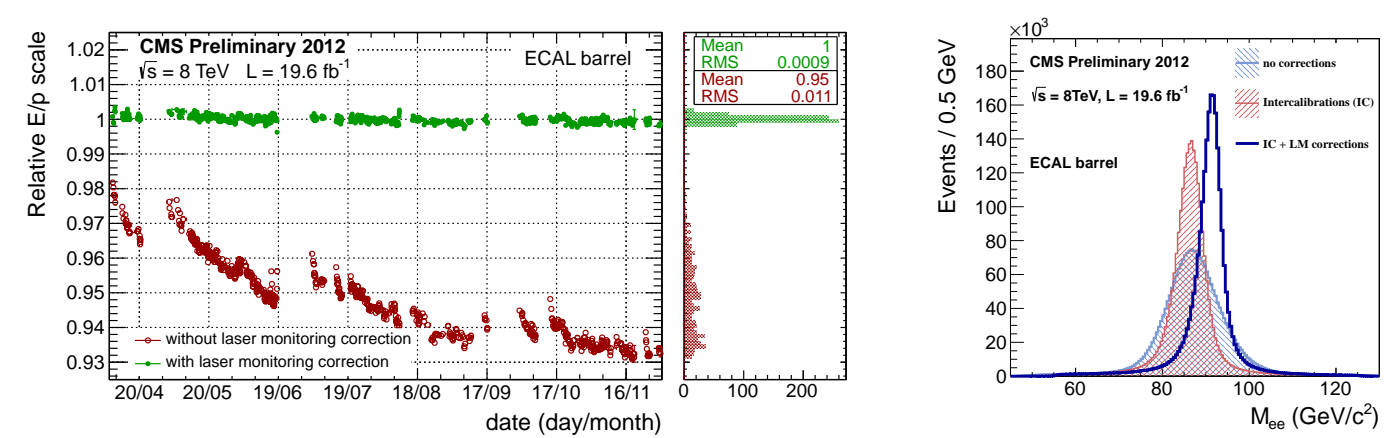

Figure 4: Left: ECAL barrel energy scale stability before (red) and after (green) laser corrections are applied, along 2012 data taking. Right: invariant mass of electrons from $\mathrm{Z}$ candidates, are shown with no corrections (light blue), crystal intercalibrations (red) and full corrections including response measured by laser monitoring (dark blue).

\subsection{Muon detectors}

Muon reconstruction in CMS is based on both the muon detectors and the tracker information. Muon tracks can be built in two directions: so called "tracker muons" consist of tracks reconstructed in the silicon tracker that are matched to hits in the muon detector, while "global muons" are muon candidates reconstructed in the muon detector that are matched inwards with a track in the tracking system. The muon identification efficiency is measured with tag-and-probe method using
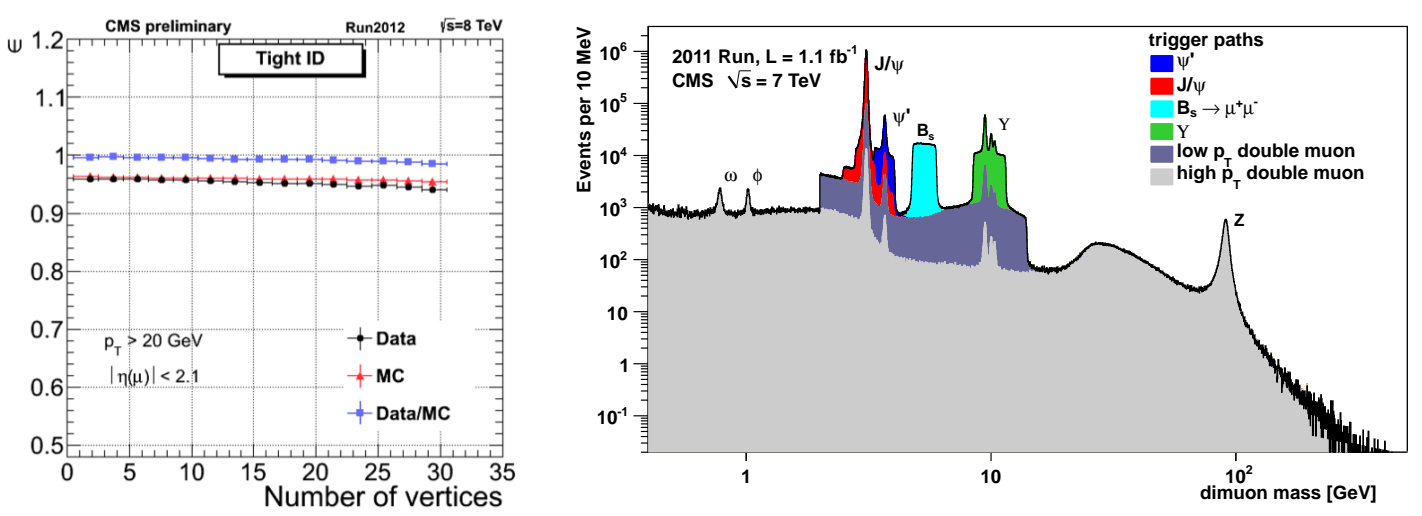

Figure 5: Left: Efficiency of offline tight muon identification as a function of the number of primary vertices. Right: Dimuon mass spectrum, divided into different HLT contributions. 
$J / \psi$ and $\mathrm{Z}$ resonances [5], and it is shown in Fig. 5 for so-called tight-muons versus the number of reconstructed vertices in the event. Tight muons are muon candidates selected with stringent quality criteria that ensure very good purity and low fake rate, while mantaining a very high efficiency, above $95 \%$. The data/montecarlo agreement is also very good. The excellent performance and flexibility of the CMS muon system can be appreciated in the right side of Fig. 5 where the dimuon invariant mass spectrum, separated in different trigger selections dedicated to the study of different resonances, is shown.

\section{Upgrade plans}

CMS has planned an extensive set of upgrades of the detector, to deal with aging and radiation damage but also to improve the performances at the very high luminosity conditions that the LHC machine is planned to deliver in next ten years, as illustrated in Fig. 6. The first long shutdown (LS1), during 2013-2014, marks the end of Run1; during Run2, in 2015-2017, the LHC is expected to produce collisions at a center-of-mass energy of 13 or $14 \mathrm{TeV}$, reaching the design luminosity of $10^{34} \mathrm{~cm}^{-2} \mathrm{~s}^{-1}$ or even more. Further machine upgrades during LS2, planned for 2018, will lead to another factor of $\sim 2$ increase in luminosity, while a more radical change is expected in LS3 (20222023) leading to High Luminosity LHC (HL-LHC), where up to 10 times the design luminosity will be achieved.

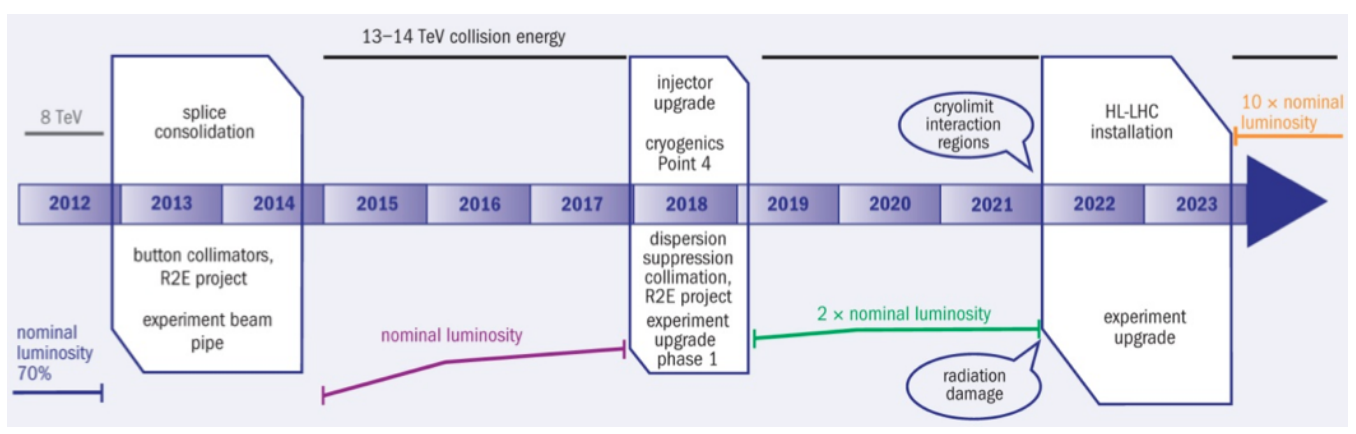

Figure 6: Plans of LHC shutdowns and related upgrades from 2012 to 2023.

\subsection{CMS Phase-1 upgrades}

Phase-1 upgrades [7] are planned for the period spanning from LS1 to LS3. During LS1, together with maintenance tasks, CMS will start several upgrade activities. The photodetectors in the hadron forward and outer calorimeters will be replaced, with multi-anode PMTs and silicon PMs; this upgrade will be extended to the barrel and endcap calorimeter during LS2, leading to improved background rejection, better particle identification and calibration, thanks to the higher gain and increased granularity. The fourth muon endcap station will be completed with CSC and RPC detectors. The electronics of the muon system, including DT electronics, will be consolidated and prepared for the Level-1 trigger upgrade, which is planned to be commissioned during Run 2 and fully deployed during the winter shutdown of 2016-2017. The new Level-1 trigger will exploit new electronics to perform better identification and measurements of trigger objects, to keep the 
lowest possible thresholds in the higher luminosity scenario offered by LHC in the next years. Also during LS1, a new, smaller (45 mm diameter) beampipe will be installed, to ease the replacement of the current pixel detector, which will happen during or before LS2. The new pixel detector, shown in Fig. 7 together with present detector for comparison, will have four barrel layers with smaller inner diameter and three endcap disks, with new readout electronics and a new cooling system. Despite the increased number of measurement layers, the new pixel detector will have a lower and more uniform material budget than the present one, as it is shown in Fig. 7 (right).
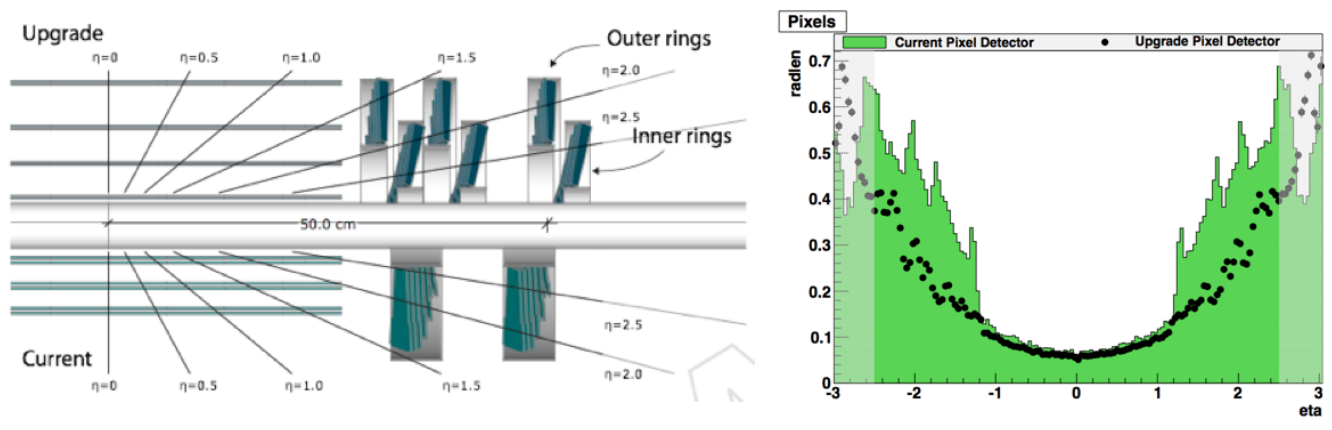

Figure 7: Left: sketch of the present and upgraded pixel detectors. Right: comparison of the material budget in the present (green histogram) and upgraded (black dots) pixel detector.

\subsection{Phase-2 upgrades}

The luminosity regime foreseen at HL-LHC will require a completely new tracking system, and a substantial redesign of the trigger and the forward calorimeter systems. Studies of a new tracking system are ongoing, targeting a technical proposal for 2014; constraints in terms of mass, power consumption, tracking capabilities in a very dense particle environment must be taken into account. Also, the idea to provide primitives for the Level-1 triggering is being investigated [8], proposing modules made of two superimposed finely-grained silicon sensors, about one mm apart. The pairs can be read out by a common device, that would be capable of correlating hits from the two sensors and discriminate the transverse momentum of such track candidates in the hardware. The response of the calorimeters at very high luminosity was simulated and no significant degradation iss expected in the barrel region, while the endcaps will require substantial efforts towards more radiation tolerant photodetectors and new scintillating materials suitable for the high radiation environment.

\section{Conclusions}

The CMS experiment has collected more than $27 \mathrm{fb}^{-1}$ during LHC run in 2010-2012, with about $93 \%$ data taking efficiency and more than $98 \%$ of the detector operational. The effort to mantain a very good detector status, together with improvements in detector understanding and the developement of more and more effective software tools, allowed excellent physics results to be delivered to the scientific community. CMS has shown the capability to face beyond-design 
pileup conditions with very good performance. The plans of upgrades to the LHC machines are new challenges for the CMS collaboration, which is preparing an extended plan of upgrades to the detector, starting with 2013-2014 shutdown and extending to and beyond 2023.

\section{References}

[1] Chatrchyan, S. and others, The CMS experiment at the CERN LHC, JINST 3, S08004, 2008.

[2] CMS Collaboration, The Trigger and Data Acquisition Project, Volume I: The Level-1 Trigger Technical Design Report, CERN/LHCC 2000-038, 2000.

[3] Bauer, G. and others, The CMS High Level Trigger System: Experience and Future Development, J. Phys.: Conf. Ser. 396 012008, 2012.

[4] CMS Collaboration, 2012 ECAL detector performance plots, CERM-CMS-DP-2013-007, 2013.

[5] CMS Collaboration, Single Muon efficiencies in 2012 Data, CMS DP 2013-009, 2013.

[6] CMS Collaboration, Pixel Performance Plots 2013, CERN-CMS-DP-2013-014, 2013.

[7] CMS Collaboration, Technical Proposal for the Upgrade of the CMS detector through 2020, CERN-LHCC-2011-006, 2011.

[8] Abbaneo, D. and others, Upgrade of the CMS tracker with tracking trigger, JINST 6, C12065, 2011. 